\title{
Rethinking the Regulation of Cellular Metabolism
}

\author{
C.B. Thompson \\ Cancer Biology and Genetics Program, Memorial Sloan-Kettering Cancer Center, \\ New York, New York 10065 \\ Correspondence: craig@mskcc.org
}

\begin{abstract}
Most biologists working today have not considered the problem of how signal transduction events, which commit cells to energetically demanding processes such as growth and division, are connected to cellular metabolism. The primary reason for this is that we have believed for the last 30 or more years that the metabolism of cells is a homeostatic, self-regulating process that does not depend on any extracellular input. The traditional view is that a mammalian cell decides to take up nutrients whenever its bioenergetic and synthetic reserves are depleted. However, a considerable body of evidence now exists that challenges the notion that the nutrient uptake and metabolism of metazoan cells are cell-autonomous.
\end{abstract}

\section{SIGNAL TRANSDUCTION ALLOWS SINGLE-CELL ORGANISMS TO DETECT EXTRACELLULAR NUTRIENTS}

First, let us consider the evolution of signal transduction. Signal transduction exists in single-cell organisms, both prokaryotes and eukaryotes. Many prokaryotic organisms have receptor kinases on their surface and the extracellular domains of those kinases are activated by nutrients the cell can use to sustain itself (for reviews, see Marijuan et al. 2010; Schaller et al. 2011). When the receptor is bound by its ligand, the intracellular domain activates its ability to act as a histidine kinase and, in most instances, phosphorylates a transcription factor, which moves to the prokaryotic DNA and initiates the transcription of an operon. The operon usually contains the genes necessary to capture that nutrient and reprogram metabolism to use the detected nutrient to maintain ATP production and to supply reduced carbon and nitrogen for macromolecular synthesis. This could well be the origin of signal transduction.

Eukaryotic organisms have evolved this paradigm even further. Yeast are constantly scavenging their environment for glucose as their preferred carbon source. However, when glucose is lacking in their environment, they switch to looking for other carbon sources by sampling their environment and scavenging what they detect. In contrast, when a source of reduced nitrogen is present, yeast will scavenge all of the glucose from their environment until none is left (for review, see Galdieri et al. 2010). There is nothing homeostatic about their glucose uptake. Some of the carbon will be used in support of exponential growth, but much of it is secreted from the cell as ethanol even in the presence of oxygen. This aerobic glycolysis seems wasteful to biochemists but is a highly efficient way to support exponential growth and to support the propagation of the species. Yeast only shift their metabolism to oxidative phosphorylation when they deplete available glucose and must turn to ethanol to maintain ATP production and viability. Only when they have run out of rich carbon sources do they switch to the efficient metabolism provided by mitochondrial oxidative phosphorylation. Oxidative metabolism is the only form of metabolism taught in a systematic way in modern biochemistry courses. It is a purely catabolic process. It is an elegant and efficient process to maximize the ATP yields when nutrients are becoming depleted and the cell seeks only to survive until nutrients are again available.

\section{MULTICELLULAR ANIMALS MUST REGULATE HOW CELLS UTILIZE SHARED NUTRIENTS}

So now let us consider the eukaryotic cells that make up multicellular animals. The vast majority of our cells engage in oxidative metabolism to maintain their survival. In fact, if any human cell acted like a yeast cell, channeling available extracellular nutrients into anabolic growth and cell division, the host's energy reserves would be rapidly depleted. This is not idle speculation - this situation is seen in hospitals on a regular basis. A yeast gets into the liver of an immunocompromised individual and the yeast rapidly discovers that it is in a nutrient-abundant environment. It immediately switches to aerobic glycolysis and exponential proliferative growth, in the process destroying the liver and killing the patient. Human cells do not behave like yeast because they have evolved a social system that prevents individual cells from promiscuously consuming the body's shared nutrient resources.

The first component of this social system was uncovered by biologists with the discovery that the constituent cells of multicellular organisms have given up the cellautonomous ability to proliferate. Human cells are not capable, in the absence of extracellular signals, of engaging in the transcription and translation of genes that can direct entry and progression through the cell cycle. However, it is increasingly clear that this alone would make for an imperfect social system (Raff 1992). For example, 
we have all had numerous viral or bacterial infections in our lifetime. During each infection, immune cells undergo a dramatic proliferative expansion leading to enlargement of the lymph nodes, tonsils, and spleen. The excess cells produced have to be eliminated at the end of the infection to restore cell number homeostasis. Excess cells die off in a process known as death by neglect, in which excess cells, no longer supported by growth factor signaling, undergo apoptosis. A default cell death program assures that cells do not accumulate in excess or in abnormal positions, an attractive addition to the social contract made by the cells that comprise metazoan organisms.

\section{DEATH BY NEGLECT DOES NOT REQUIRE APOPTOSIS}

To study death by neglect, investigators isolated cells from an intact organism by making single-cell suspensions that were cultured in medium lacking serum-derived growth factors (Rathmell et al. 2000). In such experiments, the isolated cells undergo an orderly process leading to cell death. In the first $12 \mathrm{~h}$, each of the cells loses the ability to take up nutrients from the environment. The cell, when separated from its organ of origin, loses the ability to transcribe, translate, and place on the cell surface the transporters required for the cell to take up nutrients. One aspect of these studies came as a surprise: It appears that metazoan cells lack the cell-autonomous ability to maintain nutrient transporters on the cell surface in sufficient quantities to keep the cell alive. These findings suggest that the fundamental social contract for metazoan cells is the loss of cell-autonomous nutrient uptake.

Loss of nutrient uptake does not kill a cell instantaneously because as the cell loses nutrient transporters, it can maintain itself for a day or two using its remaining internal metabolites. However, over time a variety of stress pathways are triggered as a cell's bioenergetic reserves are depleted. These stress pathways activate the cell-killing properties of the proapoptotic members of the $\mathrm{Bcl}-2$ family, resulting in apoptotic cell death (Fig. 1). However, even if it does not undergo apoptosis, a neglected cell will still ultimately die because of its inability to take up nutrients. To prove this, we made a strain of mice deficient in Bax and Bak. The mice are capable of living well past a year without any apoptosis (Lindsten et al. 2000). The organs maintain homeostatic cell numbers because excess cells die of starvation. Killing off cells by this starvation response takes 3-4 wk longer than apoptotic death by neglect, but it is just as effective (Lum et al. 2005). The extra survival time results from the cell turning to autophagy to maintain its bioenergetics by catabolizing intracellular substrates. However, autophagy is a self-limited survival strategy; ultimately there is nothing left to catabolize in the cell. Although there is plenty of extracellular glucose and amino acids, the cell dies of bioenergetic collapse.

\section{RETHINKING THE SOCIAL CONTRACT OF METAZOAN ANIMALS}

The above findings lead to a different way of thinking about the regulation of metazoan cell survival. Every cell in your body wakes up every day not needing to be talked out of suicide, but instead needing to be told to eat. It is ligand-initiated signal transduction that gives the cell the information to take up nutrients in an orderly way and use them to maintain cell survival and effector function. Thus, there is a fundamental barrier that blocks cells from becoming transformed - their inability to take up sufficient nutrients to engage in anabolic metabolism (Fig. 2). If nutrient uptake is controlled by signaling pathway(s), a cell must acquire mutations that render it cellautonomous for nutrient uptake to become a successful cancer cell. It is receptor tyrosine kinases that initiate the ability of a cell to place glucose transporters on their surface and initiate the capture of glucose and its catabolism via the glycolytic pathway. This receptor-initiated glucose uptake can be in excess of the cell's need for glucose. Glucose uptake is not homeostatic; instead, ligand-stimulated receptor signal transduction instructs mammalian cells to take up glucose, and signaling strength determines the quantity of glucose the cell consumes. If that uptake exceeds the cell's needs, the cell will have, at all times, a higher ATP/ADP ratio. The excess nutrient catabolism will saturate the mitochondria's

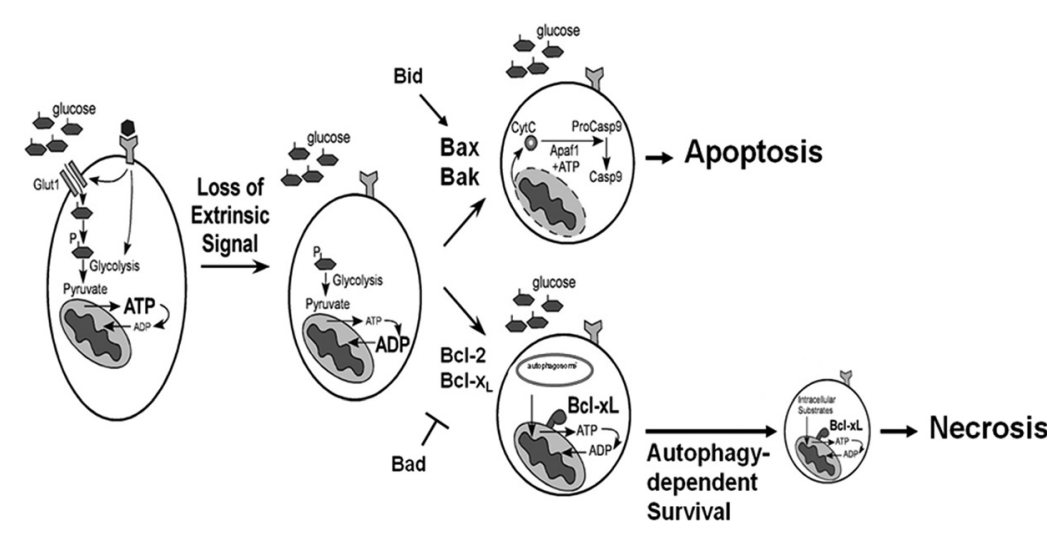

Figure 1. Unlike single-cell organisms, mammalian cells are unable take up nutrients in the absence of growth-factor-initiated instruction. 
A Growth Signal

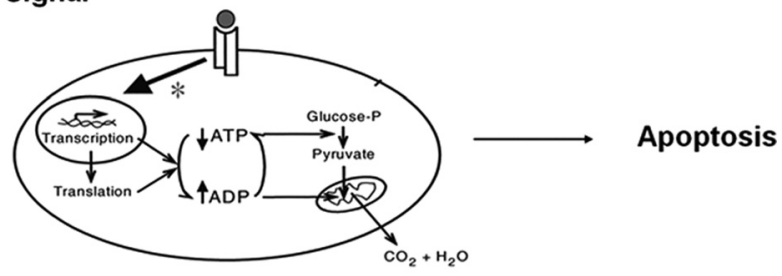

B Fuel Signal

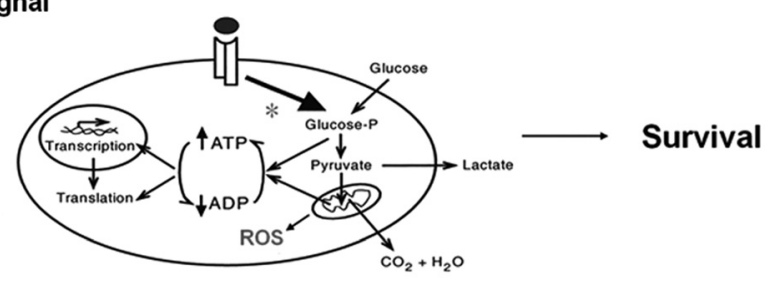

Fenton Reaction: $\mathrm{H}_{2} \mathrm{O}_{2}+\mathrm{Fe}^{2+} \rightarrow \mathrm{OH}+\mathrm{OH}-+\mathrm{Fe}^{3+}$

Figure 2. Optimal cell proliferation requires two signals. $(A)$ Cells transformed with only an activated component of growth signaling die. $(B)$ Growth-factor-enhanced fuel signaling promotes cell survival accompanied by reactive oxygen species (ROS) production and DNA mutation.

electron transport chain and the cell will start to accumulate reactive oxygen species (ROS). At that point, the cell has the energy reserves to sustain transcription and translation and is resistant to apoptosis because its mitochondria are substrate rich. The cost of this excess in bioenergetic substrates is increased production of hydrogen peroxide, a perfect first step to cancer. The cell is cell-autonomous for survival, it is eating more than it needs, and it is mutagenizing itself. Thus, the cell over time has the ability to select other mutations that will allow it to enter the cell cycle cell-autonomously.

Receptor tyrosine kinases activated by protein ligands instruct cells to pick up more glucose than they need. The best known is the insulin receptor, which is activated by a protein ligand called insulin. The insulin receptor kinase can act through adaptor proteins to activate PI3K to produce PIP3. PIP3 in turn brings to the membrane a serine threonine kinase, Akt, that activates the ability of cells to take up glucose. This is accomplished by controlling glucose transporter surface expression and enhancing the cell's ability to capture glucose by hexokinase-dependent phosphorylation of glucose, then activating phosphofructokinase to commit the captured glucose to catabolism. That is the pathway by which all professional metabolic tissues respond to insulin. They take up more glucose then they need and store it for a rainy day; the liver stores it by making glycogen first and then fatty acids, the muscle stores it by taking glucose up and storing it as glycogen, and fat cells store glucose-derived fat.

\section{CANCER AS A CONSEQUENCE OF CELL- AUTONOMOUS NUTRIENT UPTAKE}

It is interesting that the three tissues included in organismal energy storage are remarkably resistant to the pathogenesis of cancer. There is virtually no basal rate of cancer in those three tissue types. Some might view the liver as an exception to this statement. However, in the absence of hepatitis $\mathrm{B}$, hepatitis $\mathrm{C}$, or exogenous carcinogens, the liver is remarkably resistant. The tissues that develop cancer are those that have no storage capacity for the extra glucose that they are instructed to take up. As a result, such cells have to catabolize glucose-derived pyruvate in the mitochondria, creating an increase in mitochondrial ROS. Cells deal with this redox stress using various cell-intrinsic defense systems. The ultimate protection, though, is activating the cell's ability to secrete excess glucose-derived carbon back into the extracellular space as lactate. That is what happens in epithelial, neuronal, and hematopoietic cells. They cannot store extra glucose and so, when they acquire a mutation that directs excess glucose uptake, they must convert to aerobic glycolysis to protect themselves. The ability to secrete excess carbon and have it cleared by the vasculature as lactate has been shown to result from ROS-mediated induction of HIF-1 $\alpha$-dependent transcription of lactate dehydrogenase-A (LDH-A), pyruvate dehydrogenase kinase (PDHK), and vascular endothelial growth factor (VEGF) (Fig. 3) (Lum et al. 2007). However, the increase in $\mathrm{H}_{2} \mathrm{O}_{2}$ produced as a result of dealing with excess glucose catabolism can enhance DNA damage and mutation.

When individual cell types were investigated for the receptors that controlled glucose uptake, most tissues were found to use lineage-specific receptors to engage in the same signaling pathway that the insulin receptor directs (Elstrom et al. 2004). This signaling ends in the same effectors; it is just not initiated by insulin. Recently, cancer genome sequencing efforts have validated the importance of this signaling pathway in cancer. Cancer sequencing led to the discovery that the catalytic subunit of PI3 kinase is one of the most common activating mutations observed in spontaneous human cancer (Samuels et al. 2004). About 25\%-30\% of all human epithelial cancers have mutations in the catalytic subunit of PI3K, 


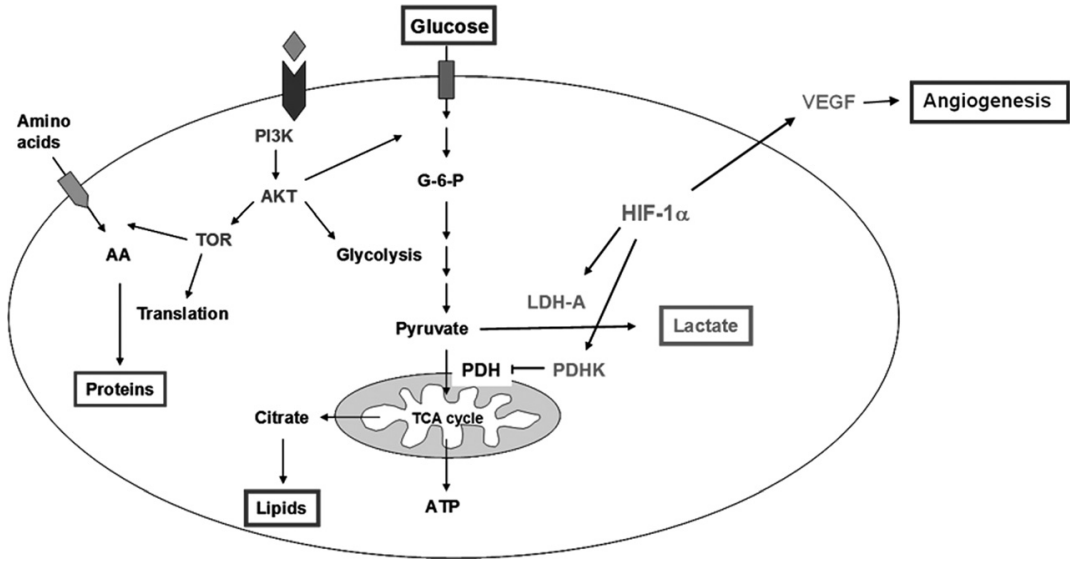

Figure 3. The PI3K/AKT/TOR pathway fuels cell growth by directing glucose uptake and metabolism. ROS-mediated HIF-1 $\alpha$ induction allows cells to eliminate glycolytic pyruvate that exceeds the cell's ability to use it for macromolecular synthesis or oxidative phosphorylation. (G-6-P) Glucose 6-phosphate, (AA) amino acids.

and these mutations direct increased glucose uptake in the cells that sustain these mutations. Subsequently, it has been shown that the second most commonly lost tumor suppressor is PTEN, the negative regulator of this pathway ( $\mathrm{Li}$ et al. 1997). When amplification of Akt and mutations in the tyrosine kinases that initiate this signaling are included, the mutation of this pathway is more than $90 \%$ in the major cancer types.

The fact that glucose uptake is deregulated in most cancers is the basis for the use of 18-fluoro-deoxyglucose uptake in cancer imaging using positron emission tomography (PET) (Vander Heiden et al. 2009). A patient is given an intravenous injection of fluoro-2-deoxyglucose (FDG) and PET imaging is used to determine where the glucose is accumulating. Following FDG injection, PET scanning allows us to image where in the body glucose is being taken up. The brain, liver, and heart are readily imaged, but most cancers take up glucose even more avidly than these tissues. This is not because the tumor is hypoxic; the only way the FDG gets to the tumor is through the vascular system, so oxygen is getting there as well. FDG accumulates in cancers because they acquire mutations that activate components of the insulin signaling pathway.

\section{AEROBIC GLYCOLYSIS PROVIDES A GROWTH ADVANTAGE TO PROLIFERATING CELLS}

Why are mutations that induce glucose uptake advantageous to tumors? Yeast have always known the answer-if they are shown a lot of glucose, they take it all up because it fuels their ability to perpetuate themselves. A high glycolytic flux allows even single-cell eukaryotes to reprogram their mitochondria in support of anabolic growth. This is the fundamental basis of the ancient endosymbiosis of mitochondria within all eukaryotic cells. There are many eukaryotic species whose mitochondria lack the ability to carry out oxidative phosphorylation, but all require mitochondria and depend on mitochondrial anabolic biosynthesis. Once a cell is flooded with glucose and there are excess precursors, mitochondria can be reprogrammed into synthetic organelles. An example of this is the production of citrate in excess of what is needed to support the tricarboxylic acid (TCA) cycle. It is exported by the mitochondria as a precursor for the synthesis of the lipids that the cell will need to produce two viable daughter cells (Fig. 3). Mitochondrial-derived citrate is needed for the production of isoprenoids, phospholipids, and the acetyl-coenzyme A needed for acetylation of proteins (Wellen et al. 2009). In the cytosol, citrate is converted into metabolic building blocks by ATP-citrate lyase (ACL), an enzyme activated downstream from Akt. The cleavage of citrate by ACL also prevents feedback inhibition of glycolysis, allowing aerobic glycolysis to proceed unchecked in support of macromolecular synthesis.

Downstream from this RTK/PI3K/Akt activation of glycolytic metabolism pathway is the activation of target of rapamycin (TOR). Akt activates TOR both directly and indirectly. TOR activation then completes the reprogramming of the cell for anabolic synthesis. TOR activation redirects amino acids into protein synthesis by increasing tRNA charging and stimulating translational initiation. The resulting increase in protein synthesis allows the cell to complete its metabolic reprogramming in support of cell growth. Constitutive activation of the PI3K/Akt/ TOR pathway leads the cell to increase in size at all stages of the cell cycle. However, whereas cell growth is optimized, the ability to proliferate requires additional metabolic resources. To engage in unbridled proliferation, mammalian cells require access to substrates in addition to glucose, in particular a supply of reduced nitrogen.

\section{GLUTAMINE IS A CRITICAL NUTRIENT FOR CELL GROWTH AND PROLIFERATION}

Critical remaining questions are, what is the source of nitrogen that supports mammalian cell proliferation and how is that source regulated by known signal transduction 


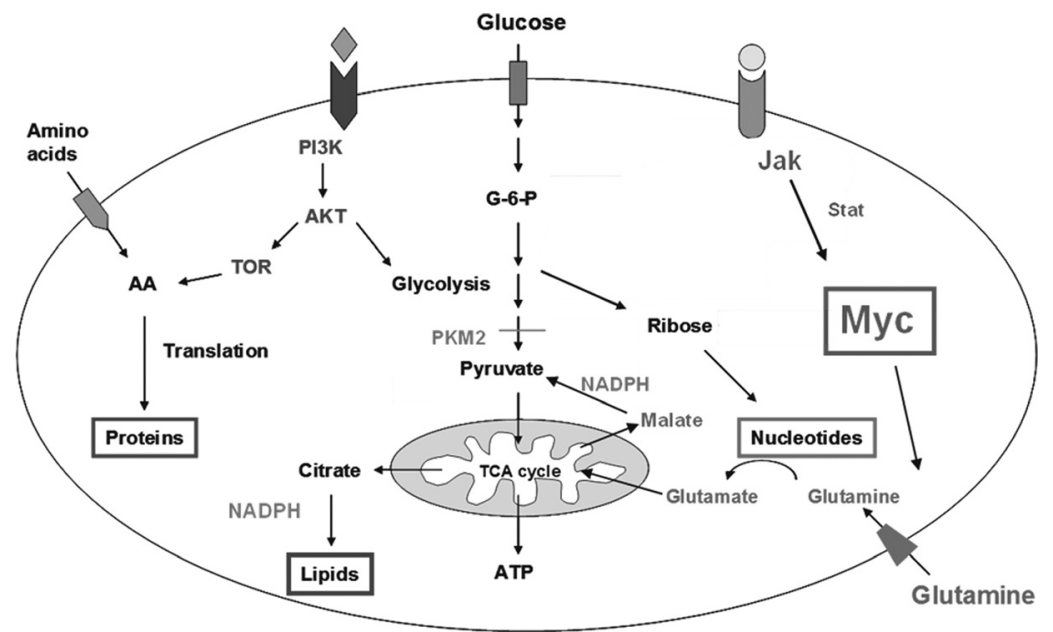

Figure 4. Myc-induced glutamine uptake and metabolism plays a key role in cell proliferation.

or oncogenes? The major source of reduced nitrogen turns out to be glutamine (DeBerardinis et al. 2007). Glutamine is normally present at $0.5 \mathrm{mM}$ in extracellular fluid and can provide both amide and imide nitrogen for synthetic reactions. Like glucose, mammalian cells lack the cell-autonomous ability to take up glutamine. However, proliferating cells depend on glutamine, and signaling pathways that initiate glutamine uptake are beginning to be defined. The regulation of glutamine uptake involves a number of upstream signaling components, but the primary effector is the transcription factor Myc (Fig. 4) (Wise et al. 2008). Through its transcriptional properties, Myc activation stimulates a cell to take up glutamine in excess of its need as an amino acid. In this seemingly wasteful process, glutamine is used to supply the TCA cycle with intermediates derived from glutamine rather than glucose metabolism. In addition, glutamine provides the cell with a nitrogen source for nucleotide and amino acid biosynthesis. However, we now believe the most critical component that glutamine provides in support of growth/proliferation is a mechanism to produce a large amount of the NADPH for macromolecular synthesis of nucleotides and fatty acids. Myc-induced glutaminolysis is also associated with a reprogramming of glucose metabolism. Myc-dependent alternative splicing of pyruvate kinase from the PKM1 isoform to PKM2 results in a slowdown of the glycolytic pathway (Clower et al. 2010; David et al. 2010). With glutamine in place as an alternative bioenergetic substrate, the cell can reprogram glycolytic metabolism to support ribose, glycerol, and serine/glycine biosynthesis, thus supplying additional substrates needed for cell proliferation. This reprogramming also coordinates the use of a glutamine as a source of nitrogen for nonessential amino acids.

This apparent excessive use of glutamine by proliferating cells is now referred to as glutaminolysis. When Myc-induced glutaminolysis was first characterized (Wise et al. 2008), the following pathway was proposed: Extracellular glutamine is taken up through ASCT2, which is a Myc-regulated gene; it is then stripped of its nitrogen, and enters the TCA cycle as $\alpha$-ketoglutarate $(\alpha \mathrm{KG}) . \alpha \mathrm{KG}$-derived malate is then exported from the mitochondria and converted by malic enzyme to pyruvate. The resulting pyruvate is then converted by LDHA (a well-characterized Myc target) to lactate and secreted from the cell (Fig. 5). In cells that display glutaminolysis, $65 \%-75 \%$ of the carbon taken up as glutamine is thrown away as lactate. The high flux of glutamine results in net NADPH production and retention of reduced nitrogen for production of nonessential amino acids and for nucleotide biosynthesis.

However, one aspect of this basic model of glutaminolysis turned out to be wrong. The NADPH that the cell

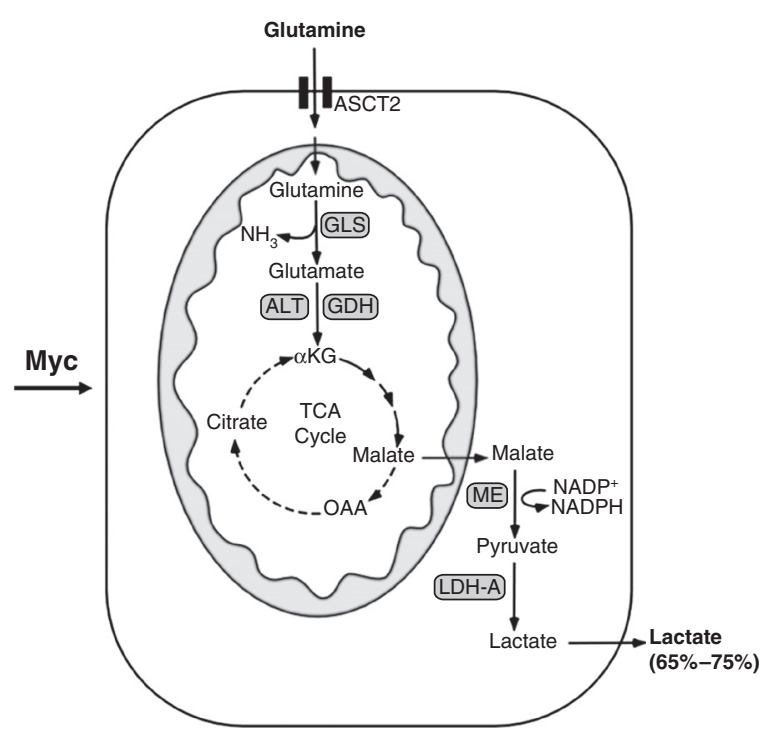

Figure 5. Myc-transformed cells metabolize glutamine in a manner that optimizes NADPH production rather than ATP production. (ASCT2) Sodium-dependent neutral amino acid transporter type 2, (GLS) glutaminase, $\left(\mathrm{NH}_{3}\right)$ ammonia, (ALT) alanine aminotransferase, (GDH) glutamate dehydrogenase, (OAA) oxaloacetate, (ME) malic enzyme, (NADP) nicotinamide adenine dinucleotide phosphate, (NADPH) reduced form of NADP. 


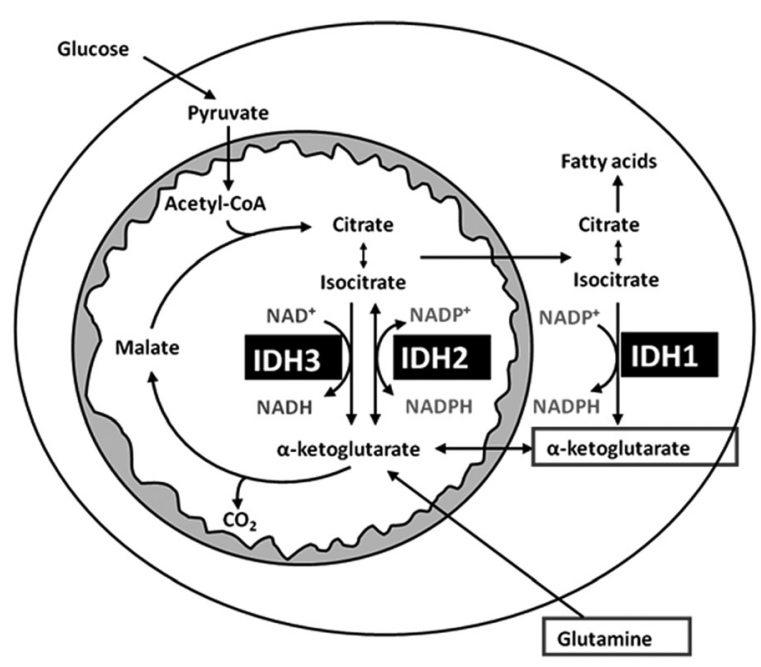

Figure 6. IDH1 and IDH 2 interconvert isocitrate and $\alpha$ ketoglutarate.

needed for anabolic growth had to be in the cytoplasm. But the Myc-regulated form of malic enzyme turned out to be mitochondrial. This created a problem for the model. NADPH is not known to be shuttled between the mitochondrial matrix and the cytosol. Thus glutaminolysis provided cells with a robust way to produce NADPH, which was the limiting source of electrons for synthetic reactions, but the NADPH was being produced in the wrong compartment, the mitochondrial matrix. Investigating this problem led to the characterization of an interesting pair of genes, isocitrate dehydrogenase 1 and 2 (IDH1 and IDH2) (Ward et al. 2010). They are not the NADH-dependent isocitrate dehydrogenase, IDH3, which is part of the TCA cycle. IDH1 and IDH2, which are not related to IDH3, catalyze a reaction that is dependent on NADPH. Furthermore, IDH1 localizes to the cytosol, whereas IDH2 localizes to the mitochondrial matrix (Fig. 6). Under the conditions that were observed in the mitochondrial matrix, there was the potential for IDH 2 to undertake a reverse reaction, converting $\alpha-K G$ to isocitrate. Isocitrate (or its isomer citrate) could then be exported from the mitochondria. The bioenergetic conditions in the cytosol should favor reconversion of isocitrate to $\alpha \mathrm{KG}$, resulting in the transfer of one NADPH equivalent from the mitochondrial matrix to the cytosol. If we depleted either IDH1 or IDH2 by RNAi in a cell displaying glutaminolysis, the cell could no longer proliferate effectively. These results suggested that the properties of IDH1 and IDH2 provided a potential mechanism by which NADPH, produced in the mitochondrial matrix, could be shuttled to the cytoplasm in quantities that may be sufficient to support anabolic synthesis of lipids and nucleic acids.

\section{CONCLUSIONS}

There is clearly a great deal more to be learned about the interrelation of glucose and glutamine metabolism in support of cell growth and proliferation, and what has been presented here is at best a first approximation of how nutrient metabolism is coordinated to support successful cell growth/proliferation. It is likely that the regulation of many other aspects of cellular metabolism will also ultimately be linked to signal transduction. The role of metabolic regulation in support of cell growth and proliferation is surprisingly still in its infancy. The wondrous catabolic cascade discovered by last century's biochemists supplies much of our understanding of how a cell sustains itself. However, it will take a much deeper understanding of metabolism to understand how a cell replicates itself. That chapter is just starting to be written.

\section{ACKNOWLEDGMENTS}

C.B.T. thanks the members of the Thompson Laboratory, past and present, for the contributions to the ideas and concepts presented in this chapter. Where the ideas presented are proven valid, it is due to their willingness to explore; where the concepts are shown to be incorrect, the fault lies with the author. In addition, the author thanks the members of the laboratory for contributing to the figures, in particular David Plas, Patrick Ward, and David Wise.

\section{REFERENCES}

Clower CV, Chatterjee D, Wang Z, Cantley LC, Vander Heiden MG, Krainer AR. 2010. The alternative splicing repressors hnRNP A1/A2 and PTB influence pyruvate kinase isoform expression and cell metabolism. Proc Natl Acad Sci 107: 1894-1899.

David CJ, Chen M, Assanah M, Canoll P, Manley JL. 2010. HnRNP proteins controlled by c-Myc deregulate pyruvate kinase mRNA splicing in cancer. Nature 463: 364-368.

DeBerardinis RJ, Mancuso A, Daikhin E, Nissim I, Yudkoff M, Wehrli S, Thompson CB. 2007. Beyond aerobic glycolysis: Transformed cells can engage in glutamine metabolism that exceeds the requirement for protein and nucleotide synthesis. Proc Natl Acad Sci 104: 19345-19350.

Elstrom RL, Bauer DE, Buzzai M, Karnauskas R, Harris MH, Plas DR, Zhuang H, Cinalli RM, Alavi A, Rudin CM, et al. 2004. Akt stimulates aerobic glycolysis in cancer cells. Cancer Res 64: 3892-3899.

Galdieri L, Mehrotra S, Yu S, Vancura A. 2010. Transcriptional regulation in yeast during diauxic shift and stationary phase. OMICS 14: 629-638.

Li J, Yen C, Liaw D, Podsypanina K, Bose S, Wang SI, Puc J, Miliaresis C, Rodgers L, McCombie R, et al. 1997. PTEN, a putative protein tyrosine phosphatase gene mutated in human brain, breast, and prostate cancer. Science 275: $1943-$ 1947.

Lindsten T, Ross AJ, King A, Zong WX, Rathmell JC, Shiels HA, Ulrich E, Waymire KG, Mahar P, Frauwirth K, et al. 2000. The combined functions of proapoptotic Bcl-2 family members bak and bax are essential for normal development of multiple tissues. Mol Cell 6: 1389-1399.

Lum JJ, Bauer DE, Kong M, Harris MH, Li C, Lindsten T, Thompson CB. 2005. Growth factor regulation of autophagy and cell survival in the absence of apoptosis. Cell 120: $237-$ 248.

Lum JJ, Bui T, Gruber M, Gordan JD, DeBerardinis RJ, Covello KL, Simon MC, Thompson CB. 2007. The transcription factor HIF-1 $\alpha$ plays a critical role in the growth factor-dependent regulation of both aerobic and anaerobic glycolysis. Genes Dev 21: $1037-1049$. 
Marijuan PC, Navarro J, del Moral R. 2010. On prokaryotic intelligence: Strategies for sensing the environment. Biosystems 99: 94-103.

Raff MC. 1992. Social controls on cell survival and cell death. Nature 356: $397-400$.

Rathmell JC, Vander Heiden MG, Harris MH, Frauwirth KA, Thompson CB. 2000. In the absence of extrinsic signals, nutrient utilization by lymphocytes is insufficient to maintain either cell size or viability. Mol Cell 6: 683-692.

Samuels Y, Wang Z, Bardelli A, Silliman N, Ptak J, Szabo S, Yan H, Gazdar A, Powell SM, Riggins GJ, et al. 2004. High frequency of mutations of the PIK3CA gene in human cancers. Science 304: 554.

Schaller GE, Shiu SH, Armitage JP. 2011. Two-component systems and their co-option for eukaryotic signal transduction. Curr Biol 21: R320-R330.
Vander Heiden MG, Cantley LC, Thompson CB. 2009. Understanding the Warburg effect: The metabolic requirements of cell proliferation. Science 324: 1029-1033.

Ward PS, Patel J, Wise DR, Abdel-Wahab O, Bennett BD, Coller HA, Cross JR, Fantin VR, Hedvat CV, Perl AE, et al. 2010. The common feature of leukemia-associated IDH1 and IDH2 mutations is a neomorphic enzyme activity converting $\alpha$-ketoglutarate to 2-hydroxyglutarate. Cancer Cell 17: 225-234.

Wellen KE, Hatzivassiliou G, Sachdeva UM, Bui TV, Cross JR, Thompson CB. 2009. ATP-citrate lyase links cellular metabolism to histone acetylation. Science 324: 1076-1080.

Wise DR, DeBerardinis RJ, Mancuso A, Sayed N, Zhang XY, Pfeiffer HK, Nissim I, Daikhin E, Yudkoff M, McMahon $\mathrm{SB}$, et al. 2008. Myc regulates a transcriptional program that stimulates mitochondrial glutaminolysis and leads to glutamine addiction. Proc Natl Acad Sci 105: 18782-18787. 


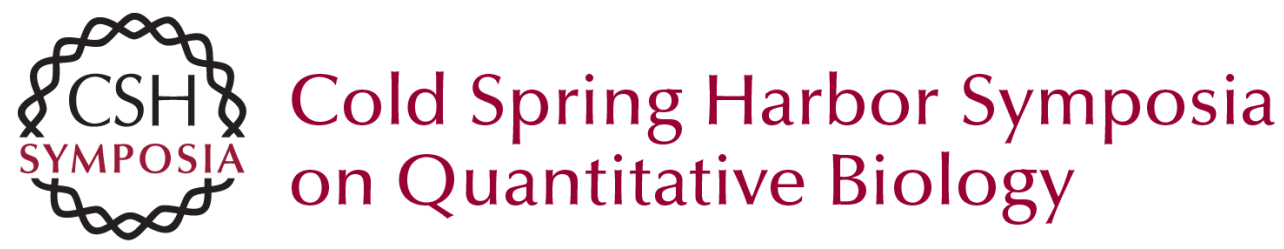

\section{Rethinking the Regulation of Cellular Metabolism}

\section{C.B. Thompson}

Cold Spring Harb Symp Quant Biol 2011 76: 23-29 originally published online March 19, 2012 Access the most recent version at doi:10.1101/sqb.2012.76.010496

References This article cites 18 articles, 9 of which can be accessed free at: http://symposium.cshlp.org/content/76/23.full.html\#ref-list-1

\section{License}

Email Alerting Receive free email alerts when new articles cite this article - sign up in Service the box at the top right corner of the article or click here. 